\title{
In Situ TEM Heating Experiments on PVP-Capped Silver Nano-Cubes
}

\author{
Sriram Vijayan ${ }^{1,2}$, Sravan Thota $^{3}$, Jing Zhao ${ }^{2,3}$ and Mark Aindow ${ }^{1,2}$ \\ 1. Department of Materials Science and Engineering, University of Connecticut, Storrs, CT USA \\ 2. Institute of Materials Science, University of Connecticut, Storrs, CT USA \\ 3. Department of Chemistry, University of Connecticut, Storrs, CT USA
}

The accurate calibration of sample temperature for TEM heating stages is a prerequisite for reliable interpretation of data from in situ heating experiments. In a recent report, the size dependent sublimation behavior of Ag nanoparticles (NPs) exhibiting the Kelvin effect was used to determine the temperature of the membrane in contact with the NPs [1]. However, electron beam overheating of the NPs, which is directly proportional to the NP radius, could lead to an over-estimate of the sublimation temperature. As such, Ag NPs with a mono-modal particle size distribution would be ideal for in situ TEM temperature calibration experiments, because the NPs would exhibit a single well-defined sublimation temperature.

The mechanism of sublimation in Ag nano-cubes (NCs), with a mono-modal size distribution [2] was investigated previously via in situ heating experiments in the TEM. However, it was found that the sublimation of the NCs could be influenced by interactions with the carbonaceous capping shell. This shell is typically made from poly-vinyl pyrolidone (PVP), which is used as a capping agent to stabilize the $\{100\}$ planes of $\mathrm{Ag}$ and favor the formation of cuboidal NPs. A further complication arises due to the limited electronic conductivity of the capping shell, which could result in overheating of the Ag NCs under the electron beam.

Here we have compared the effects on mono-modal Ag NCs of electron beam heating and in-situ heating using a MEMS based heating stage [3] (Nano Ex- i/V, FEI) on a 200kV TEM/STEM (Talos, F200X, FEI) with a Schottky field emitter. Examples of the data are shown in Figure 1. Under electron beam heating only, there is nucleation of new Ag grains on the surface of the single-crystal [001]-oriented NCs (Figures 1(a)-(c)), presumably due to surface diffusion of Ag underneath the capping layer. During subsequent in situ heating on the MEMS chip, however, the small Ag grains are re-absorbed by the NC, which then spheroidizes prior to sublimation. The morphological development during sublimation is different from that reported previously [2], suggesting that this may be related to the rupture of the capping shell along the sides of the NC. These data are compared with the sublimation temperature expected from the Kelvin effect to reveal the role of beam heating in the experiment. [4]

\section{References:}

[1] M.A. Asoro, D. Kovar, \& P. J. Ferreira, ACS Nano, Vol. 7, No. 9 2013, p 7844

[2] Y. Ding, F.Fan, Z. Tian, \& Z. L. Wang, Small, Vol. 5, No.24, 2009, p 2812

[3] L. Mele et al, Res. \& Technique, 2016, (doi: 10.1002/jemt.22623)

[4] This work was supported in part by a research grant from FEI Company under an FEI-UConn partnership agreement and by the award of an FEI Graduate Fellowship to Sravan Thota. The studies were performed in the UConn/FEI Center for Advanced Microscopy and Materials Analysis (CAMMA). 
(a)
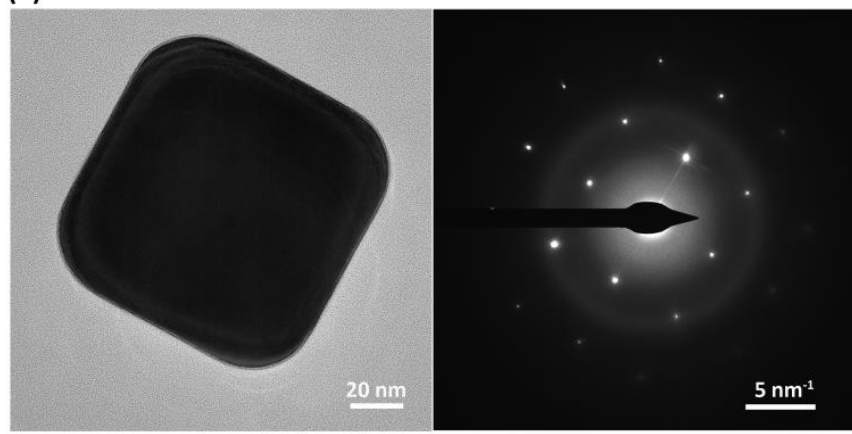

(b)

(c)

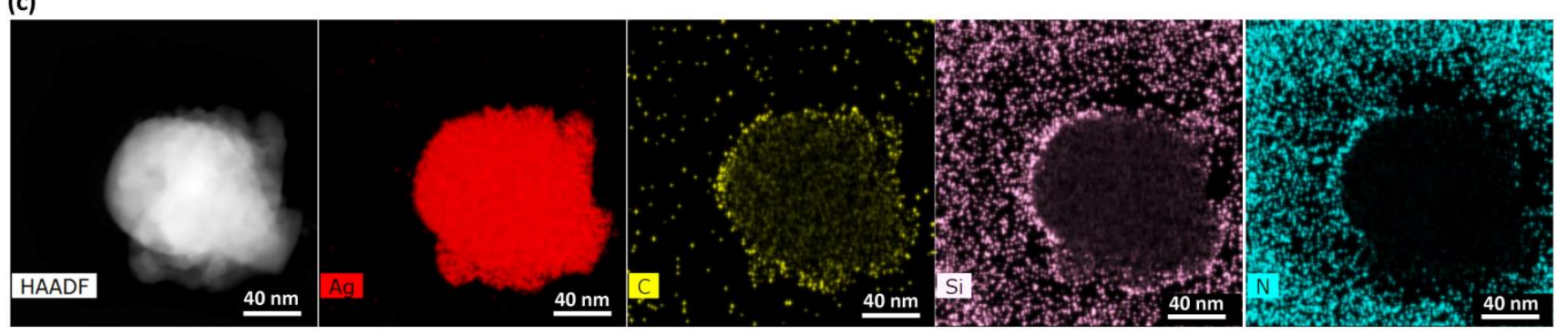

(d)
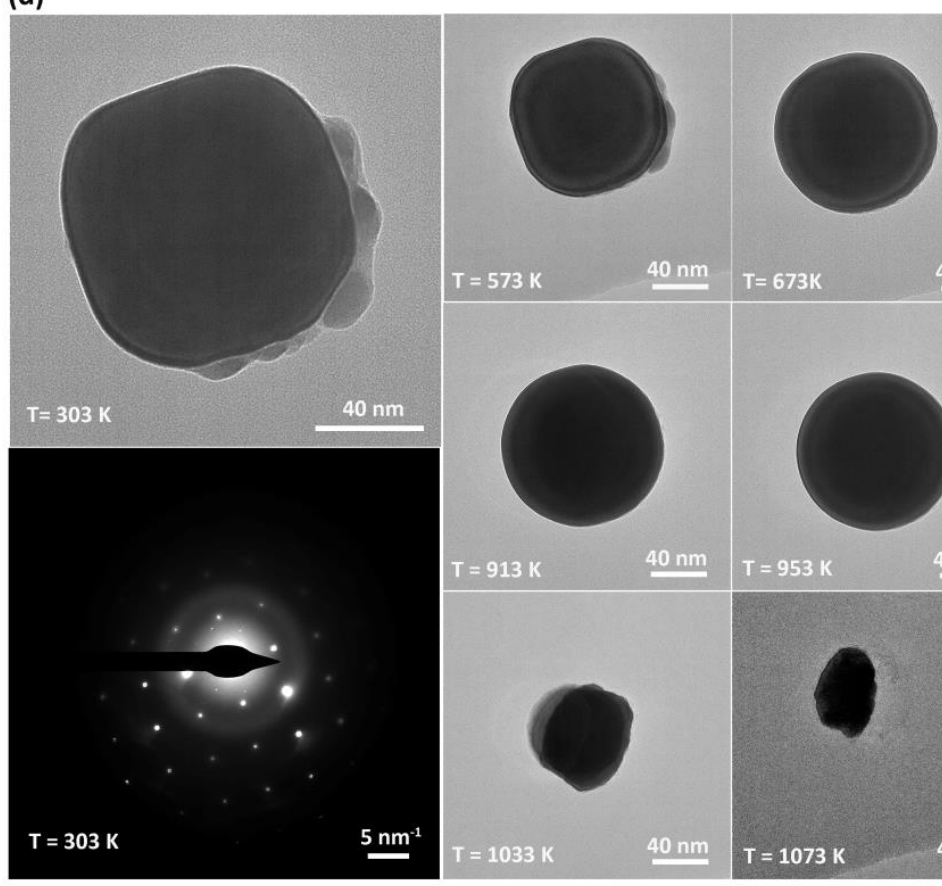

$40 \mathrm{~nm} \quad \mathrm{~T}=673 \mathrm{~K}$
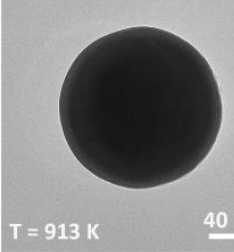

$40 \mathrm{~nm} \quad \mathrm{~T}=953 \mathrm{~K}$

$40 \mathrm{~nm} \quad \mathrm{~T}=993 \mathrm{~K}$

$40 \mathrm{~nm}$

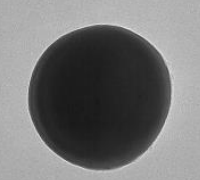

$\mathrm{T}=893 \mathrm{~K}$

$40 \mathrm{~nm}$

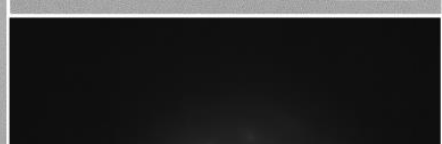

Figure 1. BF-TEM image \& SADP of (a) a [001]-oriented single crystal Ag-NC (b) the same NC after 3120s under the beam. (c) HAADF STEM image and corresponding elemental maps of an Ag NC on a $\mathrm{Si}_{3} \mathrm{~N}_{4}$ membrane after exposure to the beam. (d) In situ heating of a NC at different temperature intervals showing the onset of sublimation at $993 \mathrm{~K}$. The retention of the [001] orientation of the cube at elevated temperatures indicates a strong interaction between the NP and the capping shell, the remains of which can still be observed at $1093 \mathrm{~K}$. 